\title{
Secukinumab Associated with Acitretin in Generalized Pustular Psoriasis
}

\author{
Gisondi Paolo*, Pizzolato Miriam and Girolomoni Giampiero \\ Department of Medicine, Section of Dermatology and Venereology, Italy.
}

*Corresponding author: Gisondi Paolo, Department of Medicine, Section of Dermatology and Venereology, Italy.

Received Date: November 19, 2018

Published Date: December 12, 2018

\begin{abstract}
Generalized pustular psoriasis, the most severe form of psoriasis, is a rare variant characterized by widespread sterile pustule associated with systemic inflammation. The disease lacks of specific treatment, drugs approved for plaque psoriasis are used in the clinical practice, with variable results. We present the case of a 41-year old woman affected by a severe form of generalized pustular psoriasis, resistant to several therapies included Tumor Necrosis Factor Antagonist; in our patient we decide to use acitretin plus Secukinumab, an IL-17 inhibitor. The association was effective, achieving the complete resolution of pustulation at month-2; the result was maintained for over 14 months of follow up. No adverse events related to therapy were observed. Generalized pustular psoriasis is related to IL-36RN mutations, which lead to upregulation in IL-36 signaling; IL-36 itself could promote IL-17 pathways. Our case demonstrates that targeting IL-17 eventually associated with systemic retinoids could be a valid therapeutic option for these patients.
\end{abstract}

Keywords: Generalized pustular psoriasis; Secukinumab; Acitretin

Abbreviations: GPP: Generalized Pustular Psoriasis; IL-17: Interleukin-17; DLQI: Dermatology Life Quality Index; IL36RN: Interleukin 36 Receptor Antagonist

\section{Introduction}

Generalized pustular psoriasis (GPP) is a severe inflammatory skin disease characterized by the presence of sterile pustules covering almost the entire body and it may be associated with systemic symptoms. There is an increasing need for specific treatment options for GPP, because this skin disorder is frequently resistant to therapies indicated for chronic plaque psoriasis. Preliminary data suggest that interleukin (IL)-17 could be a promising therapeutic target for GPP.

\section{Case Presentation}

A 41 years old Moroccan woman with a 6-year history of GPP was admitted with an acute, severe flare of pustulation. On exanimation, she was febrile and had disseminated, painful pustules primarily over the back, the upper and lower extremities (Figure 1A). There was a generalized erythema covering approximately $80 \%$ of the body surface area; the dermatology life quality index (DLQI) was 21. Secukinumab $300 \mathrm{mg}$ with initial dosing at weeks 0, 1, 2 and 3, followed by monthly maintenance dosing starting at week 4 was administered in association with acitretin $0.4 \mathrm{mg} / \mathrm{Kg} /$ day. Within 2 weeks after the first secukinumab dose, there was an improvement in her general state, partial resolution of pustules and erythema. Complete GPP clearance was achieved at month-2 (Figure 1B) when DLQI score was 4 . The patient was otherwise healthy, and she was taking hormonal contraception. The beneficial was maintained during the 14 months of follow up.

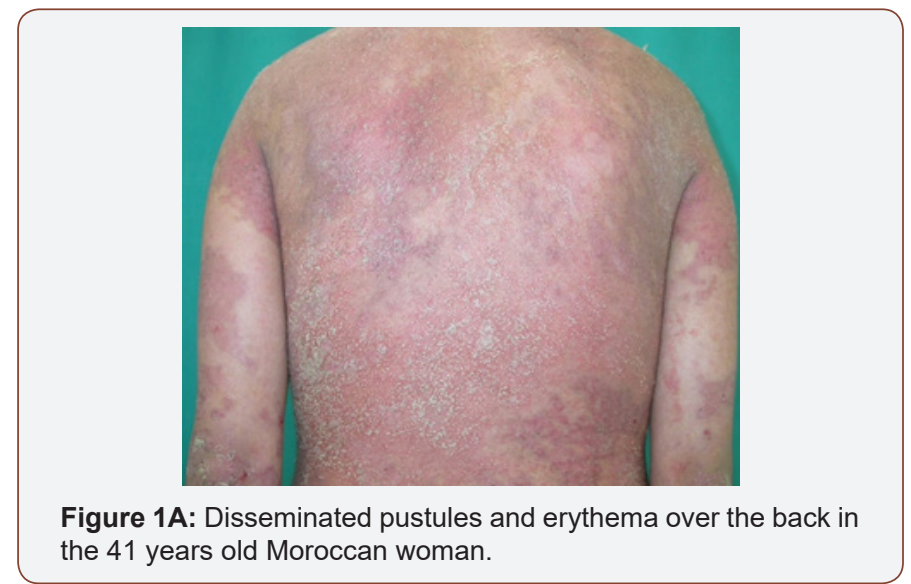




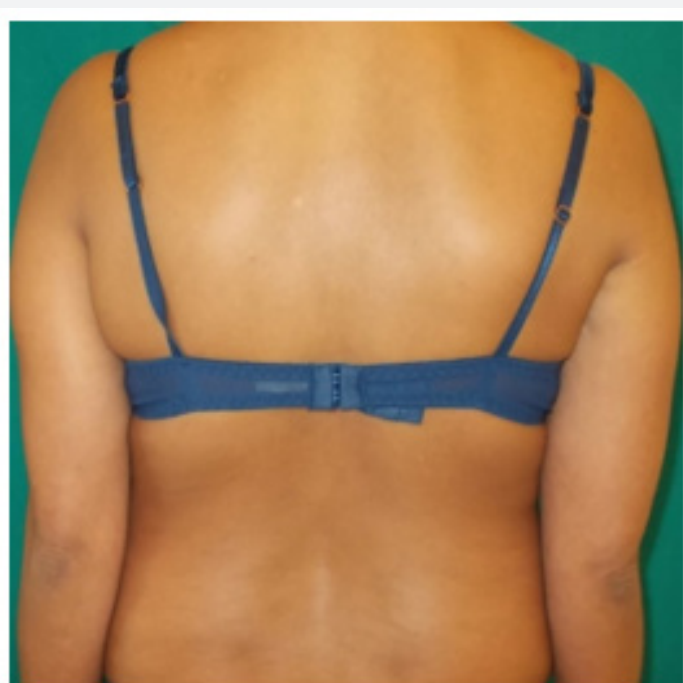

Figure 1B: Complete clearance of pustulation and erythema achieved two months after the first secukinumab administration associated to acitretin.

\section{Discussion}

Patients with GPP have higher levels of IL-17 and an increased number ofIL-17-producing cells in blood and lesional skin compared to healthy controls and patients with chronic plaque psoriasis $[1,2]$. In pustular psoriasis, IL-17 contributes to recruitment and activation of the neutrophils in the pustules [3]. Therefore, IL17 could be a relevant therapeutic target for pustular psoriasis. The effectiveness of IL-17 inhibitors secukinumab, ixekizumab \& brodalumab [4-6] in GPP has been reported in some case reports and clinical trials. In particular, in a phase III open-label multicenter study in patients with GPP $(n=12)$ disease clearance was obtained in $83.3 \%(n=10)$ of patients at week 16 treated with secukinumab [4]. Our case supports the effectiveness of secukinumab associated with acitretin in the treatment of GPP in a young female patient with a long-standing history of GPP being resistant to several systemic treatments including cyclosporine, methotrexate, infliximab and etanercept. In addition, the association with acitretin was effective and well tolerated.

In the last years the mutation of IL-36RN (IL-36 receptor antagonist) has been reported as the major pathogenic causes of familiar and sporadic cases of GPP: deficiency in IL36RN bring to an unopposed IL-36 signaling. IL-36 pathway may facilitate pathogenic Th17 responses, either directly or through the induction of IL-23 [7]. Moreover, IL-17A can induce expression of IL-36, so a feedback loop between the IL-36 and IL-17 involved in driving cytokine expression in psoriatic tissue has been described [8]. Some patients carry any mutation of this gene, but regardless of IL-36RN mutations, level of IL-36RN are lower [7]: probably defect in regulation of IL36 signaling is a common pathological pathway in GPP. A limitation of our study is that the patient was not investigated for mutation of IL-36RN gene; however, we suggest that the inhibition of IL-17 would be beneficial independent of IL36RN-gene mutation. Controlled and larger studies are needed to confirm the long-term effectiveness of IL-17 inhibitors in patients with GPP.

\section{Conclusion}

GPP is a severe inflammatory skin disease characterized by the presence of sterile pustules covering almost the entire body and systemic symptoms such as fever. The treatment of GPP is challenging because it is generally very resistant to therapies including acitretin. Secukinumab, a fully human-recombinant antiIL-17A monoclonal antibody is indicated for psoriasis vulgaris and psoriatic arthritis. Accumulating studies provide evidence of the effectiveness of IL-17 inhibitor secukinumab also in GPP, although this should be confirmed in further controlled studies. The therapeutic association between secukinumab and acitretin was an effective and safe option in our case with a severe form of GPP.

\section{Acknowledgement}

None.

\section{Conflicts of Interest}

1.Conflict of interest of Paolo Gisondi: who has been paid as consultant from AbbVie, Abiogen, Almirall, Celgene, Eli-Lilly, Janssen, Leo Pharma, Merck, MSD, Novartis, Otsuka, Pfizer, Pierre Fabre, Sanofi, UCB.

\section{Conflict of interest of Miriam Pizzolato: None.}

3. Conflict of interest of Giampiero Girolomoni: who has been paid as consultant from

AbbVie, Abiogen, Allmirall, Amgen, Bayer, Biogen, Boehringer Ingelheim, Celgene, Eli-Lilly, Galderma, Hospira, Leo Pharma, Merck, MSD, Mundipharma, Novartis, Pfizer, Pierre Fabre, Regeneron, Sandoz, Sanofi, Sun Pharma.

\section{References}

1. Song HS, Kim SI, Park TI, Jang YH, Lee ES (2016) Immunohistochemical Comparison of IL-36 and the IL-23/Th17 Axis of Generalized Pustular Psoriasis and Acute Generalized Exanthematous Pustulosis. Ann Dermatol 28(4): 451-456.

2. Yilmaz SB, Cicek N, Coskun M, Yegin O, Alpsoy E (2012) Serum and tissue levels of IL-17 in different clinical subtypes of psoriasis. Arch Dermatol Res 304(6): 465-469.

3. Kolls JK, Lindén A (2004) Interleukin-17 family members and inflammation. Immunity 21(4): 467-476.

4. Imafuku S, Honma M, Okubo Y, Komine M, Ohtsuki M, et al. (2016) Efficacy and safety of secukinumab in patients with generalized pustular psoriasis: A 52-week analysis from phase III open-label multicenter Japanese study. J Dermatol 43(9): 1011-1017.

5. Saeki H, Nakagawa H, Nakajo K, Ishii T, Morisaki Y, et al. (2017) Efficacy and safety of ixekizumab treatment for Japanese patients with moderate to severe plaque psoriasis, erythrodermic psoriasis and generalized pustular psoriasis: Results from a 52-week, open-label, phase 3 study (UNCOVER-J). J Dermatol 44(4): 355-362.

6. Yamasaki K, Nakagawa H, Kubo Y, Ootaki K, Japanese Brodalumab Study Group (2017) Efficacy and safety of brodalumab in patients with generalized pustular psoriasis and psoriatic erythroderma: results from a 52-week, open-label study. Br J Dermatol 176(3): 741-751.

7. Arakawa A, Vollmer S, Besgen P, Galinski A, Summer B, et al. (2018) Unopposed IL-36 activity promotes clonal CD4+ T-cell responses with IL-17A production in generalized pustular psoriasis. J Invest Dermatol 138(6): 1338-1347.

8. Carrier Y, Ma HL, Ramon HE, Napierata L, Small C, et al. (2011) Interregulation of Th17 cytokines and the IL-36 cytokines in vitro and in vivo: implications in psoriasis pathogenesis. J Invest Dermatol 131(12): 2428-2437. 